\title{
Correlation of telencephalic afferents and SDH distribution in the bony fish Polypterus
}

\author{
MARK R. BRAFORD, Jr. and R. GLENN NORTHCUTT
}

Division of Biological Sciences, The University of Michigan, Ann Arbor, Mich. 48109 (U.S.A.)

(Accepted March 9th, 1978)

Recent studies of Polypterus ${ }^{1}$ and other actinopterygian fishes ${ }^{3,6,9}$ have demonstrated that the olfactory bulb projects to restricted portions of the telencephalon proper. In Polypterus the major target of the olfactory bulb is the superficial portion of pallial area P1. In terms of this input and its topological position area P1 is comparable to the piriform pallium of tetrapods. Smaller olfactory targets include VI and the so-called entopeduncular complex in the subpallium. The remainder of the pallium (areas $\mathrm{P} 2$ and $\mathrm{P} 3$ ) and large parts of the subpallium are devoid of secondary olfactory afference. In an attempt to identify additional sensory areas, we have studied the regional distribution of succinate dehydrogenase (SDH) in the telencephalon of Polypterus. We selected this approach because high concentrations of SDH have been previously reported in the primary somatosensory, auditory and visual cortices of mammals ${ }^{5}$ and in regions of the dorsal ventricular ridge which receive auditory and visual projections from the thalamus in crocodiles ${ }^{8}$.

Specimens of Polypterus palmas were killed by decapitation. The brains were removed and placed in plastic embedding molds containing a water-soluble resin (Tissue-Tek Il, Lab-Tek Products, Div. Miles Laboratories, Inc., Naperville, III. 60540). Quick freeze was achieved by immersing the embedded brains in 2-methylbutane cooled to $-70^{\circ} \mathrm{C}$ in a dry ice and ethanol bath. Transverse sections were cut at $25 \mu \mathrm{m}$ on a cryostat at $-20^{\circ} \mathrm{C}$ and collected on chrome-alum treated slides. SDH activity was demonstrated by the method of Pearse ${ }^{7}$ with Nitro-BT. The most consistent results were obtained using Nitro-BT from Dajac Laboratories, Borden Chemical, Div. of Borden, Inc., Philadelphia, Pa. 19124. Incubation times were $90 \mathrm{~min}$ at $40^{\circ} \mathrm{C}$. The regional distribution of SDH was analyzed by projecting individual sections and tracing the outlines of the section and the boundaries of the reactive zones. High, moderate and low concentrations were recognized by visual inspection and their distribution is shown in Fig. 1A-C.

High concentrations of SDH are present in the glomerular layer of the olfactory bulb and in the superficial portion of pallial area PI - major targets of the olfactory nerve and olfactory bulb, respectively. In addition, the superficial portions of the nonolfactory pallial areas (P2 and P3), as well as a deeper portion of $\mathrm{P} 2$ at rostral levels, display high $\mathrm{SDH}$ concentrations, suggesting that they are sensory areas. 

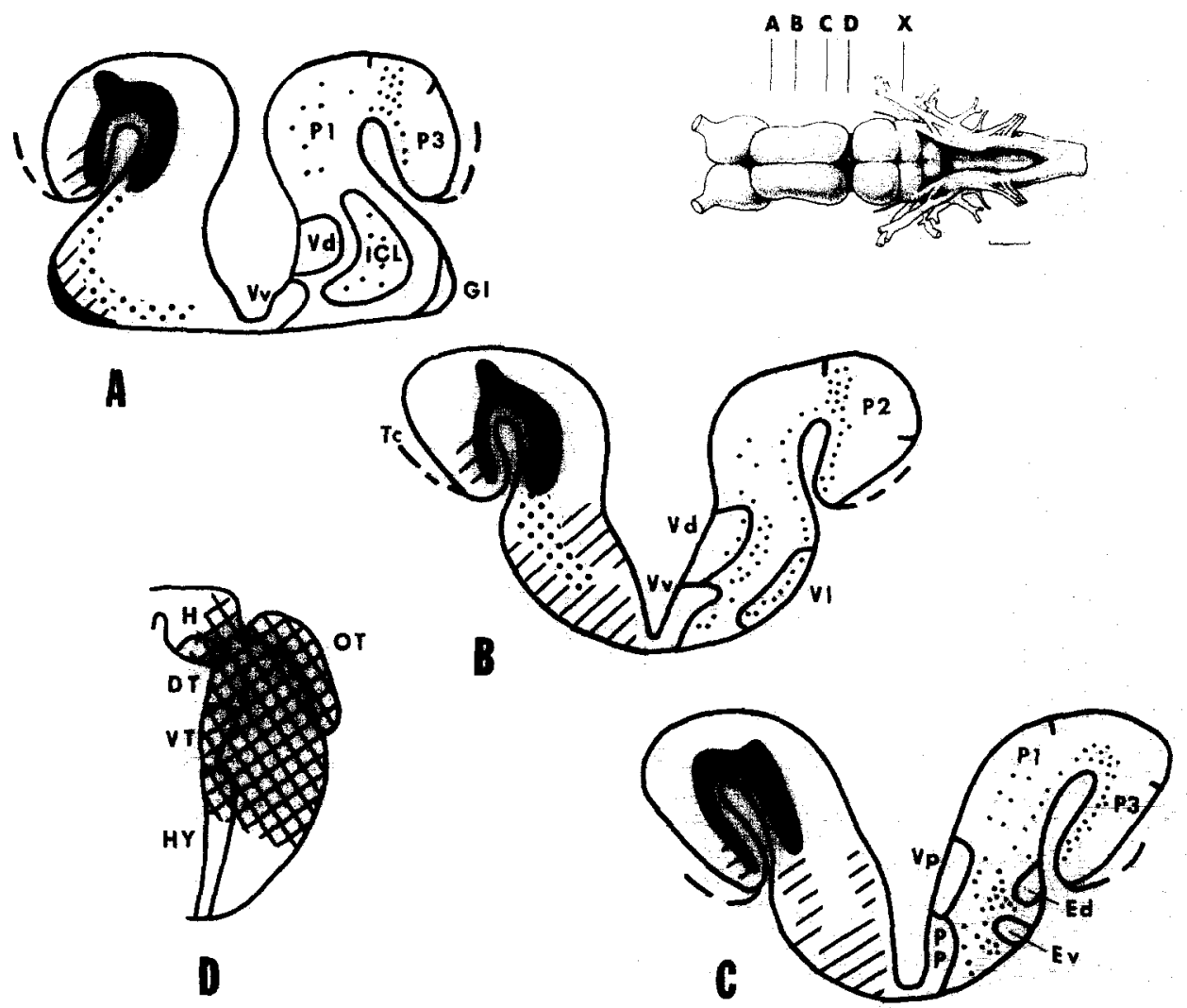

Fig. 1. Charting of SDH distribution and ascending telencephalic afferents. A-C: cross-sections of the telencephalon at levels indicated in dorsal view of brain (bar scale for dorsal view: $2 \mathrm{~mm}$ ). SDH distribution is shown on the left with solid black indicating high concentrations, hatching indicating moderate concentrations, and stippling indicating low concentrations. The distribution of terminal degeneration, following a large aspiration lesion of the ipsilateral diencephalon by an approach through the optic tectum, is plotted on the right. D: the extent of the lesion is indicated by cross-hatching at the level of the habenular commissure. $X$ in dorsal view indicates the level of control hemisection through the isthmus. Abbreviations: DT, dorsal thalamus; Ed, dorsal entopeduncular nucleus; Ev, ventral entopeduncular nucleus; Gl, glomerular layer of olfactory bulb; $\mathbf{H}$, habenula; $\mathrm{HY}$, hypothalamus; $\mathrm{OT}$, optic tectum; PP, nucleus preopticus periventricularis; $\mathrm{Pl}, \mathrm{P} 2$ and $\mathrm{P} 3$, pallial areas; Tc, tela choroidea; Vd, dorsal subpallial nucleus; Vl, lateral subpallial nucleus; $\mathrm{Vp}$, posterior| subpallial nucleus; VT, ventral thalamus; Vv, ventral subpallial nucleus.

To determine the origin of this suggested input to the non-olfactory pallium, two specimens of Polypterus palmas were subjected to large, unilateral aspiration lesions of the diencephalon by an approach through the rostral optic tectum. Following survival times of 12 and 13 days, the brains were processed by Fink-Heimer procedure $\mathrm{II}^{4}$ to demonstrate degenerating axons and their terminals. The telencephatic distribution of the degeneration in one of these cases is charted in Fig. 1A-C and the maximal extent of the lesion is shown in Fig. 1D.

A heavy terminal field is present in the superficial portion of pallial areas $\mathbf{P} 2$ and P3. At rostral levels (Fig. 1A, B) the terminals extend more deeply (dorsally) toward the ependymal surface in $\mathrm{P} 2$. Heavy terminals are also seen in the region of 

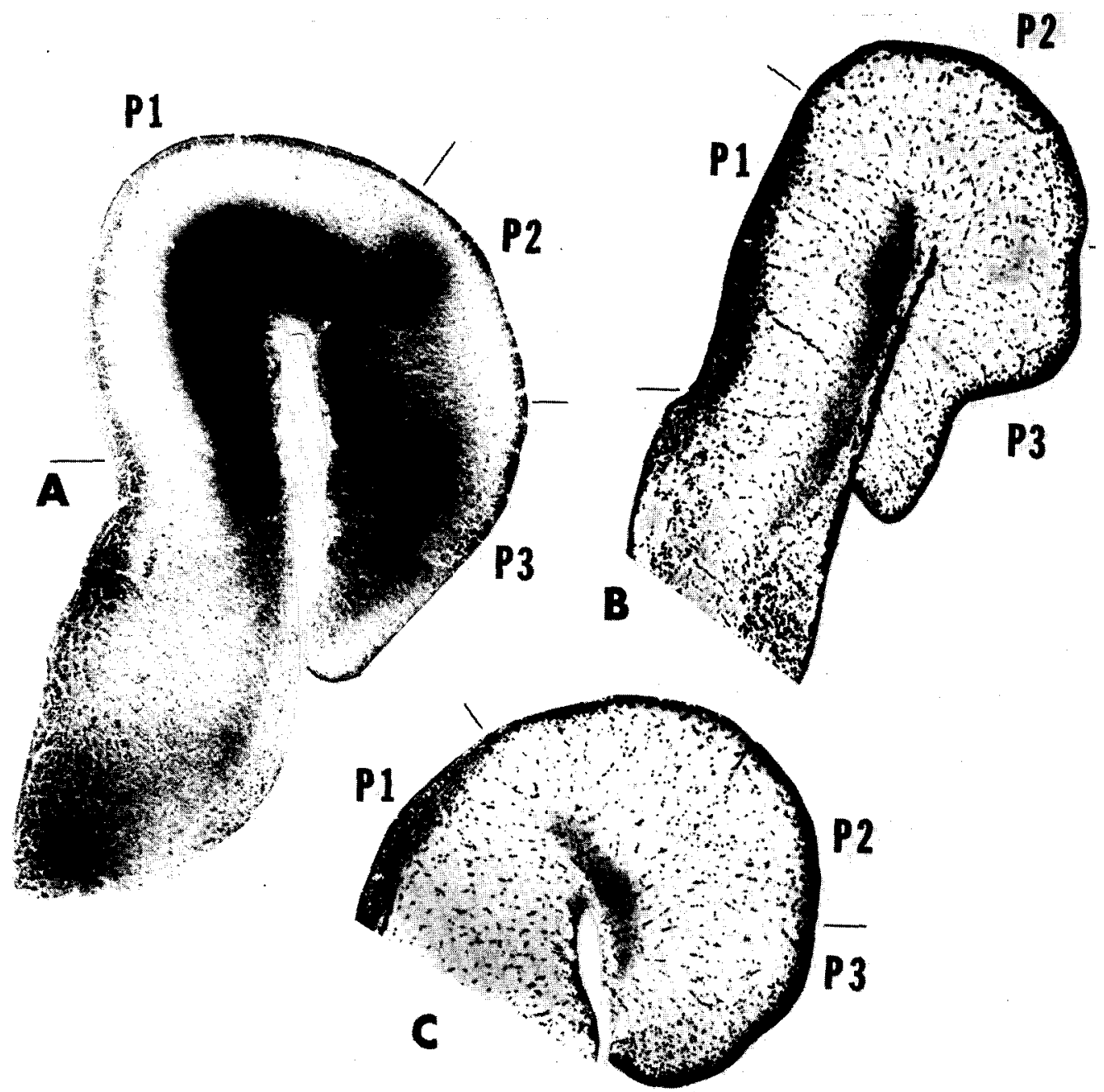

Fig. 2. Correlation of SDH distribution and telencephalic afferents. A: photomicrograph of SDH distribution. High concentrations are present in the superficial portions of all pallial zones with a deeper (more dorsal) extension in P2. Moderate concentrations are seen in the deeper (more lateral) part of P3 and in portions of the subpallium. Method of Pearse, incubation time 90 min at $40^{\circ} \mathrm{C}$. $\mathrm{B}$ : photomicrograph of terminal degeneration in pallial area $\mathrm{P} 1$ following ablation of the ipsilateral olfactory bulb. Note correspondence with band of high SDH concentration in P1. Fink-Heimer method, survival time 16 days. $C$ : photomicrograph of terminal degeneration in $\mathrm{P} 2$ and $\mathrm{P} 3$ following a large aspiration lesion of the ipsilateral diencephalon. Note correspondence with the high SDH concentrations in P2 and P3. Fink-Heimer method, survival time 12 days. Abbreviations are the same as in Fig. 1.

the entopeduncular complex in the subpallium. Moderately dense terminals are present in $\mathrm{Vl}$ and the lateral parts of $\mathrm{Vd}$ and $\mathrm{V}_{\mathrm{s}}$, whereas sparse terminals are seen in the internal cellular layer of the olfactory bulb and in the deep portion of PI. The superficially located degeneration in $\mathrm{P} 1$ represents axons of tractus pailii en route to $\mathrm{P} 2$ and $\mathrm{P} 3$ (Fig. 1 B, C).

In separate control cases unilateral tectal lesions, large electrolytic lesions of the 
tegmentum, and hemisection of the brain at the level of the isthmus (see ' $x$ ' in insert in Fig. 1) all failed to produce the degeneration in the telencephalon seen after the diencephalic lesions. Thus these telencephalic afferents appear to originate from the diencephalon.

The combined distribution of the olfactory projection to $\mathrm{P} 1$ and the ascending projection to $\mathrm{P} 2$ and $\mathrm{P} 3$ coincides in precise detail with the regions of high SDH concentration in the pallium (Fig. 2). The correspondence holds throughout the entire extent of the pallium including the 'bump' in P2 which is present rostrally but not caudally. Areas of moderate and low SDH concentration in the subpallium also correspond closely to the areas which receive olfactory and/or ascending inputs. The moderate SDH concentration in the deeper (more lateral) part of P3 suggests that this region receives a large afferent projection from an unknown source.

This is the first report of ascending projections to the telencephalon in an actinopterygian fish. Although the exact source and nature of these projections remain to be determined, we interpret their presence as prima-facie evidence for non-olfactory sensory regions in the telencephalon of Polypterus.

Our results, together with previous findings in mammals and reptiles, further suggest that SDH distributions, at least in the telencephalon, are reliable markers for sensory projection areas and may be useful as predictors in the absence of experimentally determined fiber connections.

We gratefully acknowledge Mr. Ronald G. Nicholes for his excellent histochemical preparations. This research was supported by NSF Grant GB-40134 to R.G.N. and NINDS Postdoctoral Fellowship NS 02560 to M.R.B.

I Braford, M. R., Jr. and Northcutt, R. G., Olfactory bulb projections in the bichir, Polypterus, J. comp. Neurol., 156 (1974) 165-178.

2 Braford, M. R., Jr. and Northcutt, R. G., Telencephalic afferents and SDH distribution in Polypterus, Neurosci. Abstr., 3 (1977) 89.

3 Finger, T. E., The distribution of the olfactory tracts in the bullhead catfish, Ictalurus nebulosus, J. comp. Neurol., 161 (1975) 125-142.

4 Fink, R. P. and Heimer, L., Two methods for selective silver impregnation of degenerating axons and their synaptic endings in the central nervous system, Brain Research, 4 (1967) 369-374.

5 Friede, R. L., Histochemical investigations on succinic dehydrogenase in the central nervous system. IV. A histochemical mapping of the cerebral cortex of the guinea pig, $J$. Neurochem., 5 (1960) 156-171.

6 Northeutt, R. G, and Braford, M. R., Jr., New observations on the organization and evolution of the telencephalon of actinopterygian fishes. In S. O. E. Ebbesson (Ed.), Comparative Neurology of the Telencephalon, Plenum Press, New York, in press.

7 Pearse, A. G. E., Histochemistry, Theoretical and Applied, 2nd ed., Little, Brown and Co., Boston, 1960, 998 pp.

8 Pritz, M. B. and Northcutt, R. G., Succinate dehydrogenase activity in the telencephalon of crocodiles correlates with projection areas of sensory thalamic nuclei, Brain Research, 124 (1977) $357-360$.

9 Scalia, F. and Ebbesson, S. O. E., The central projections of the olfactory bulb in a teleost (Gymnothorax funebris), Brain Behav. Evol., 4 (1971) 376-399. 